\title{
Duocarmycins binding to DNA investigated by Molecular Simulation
}

Katrin Spiegel ${ }^{1}$, Ursula Rothlisberger ${ }^{2}$ and Paolo Carloni ${ }^{3,4}$

${ }^{1}$ University of Pennsylvania, Center for Molecular Modeling, Philadelphia, PA 19104

${ }^{2}$ EPFL, Ecole polytechnique federale de Lausanne, Laboratory of Computational Chemistry and Biochemistry, 1015 Ecublens, $\mathrm{CH}$

${ }^{3}$ SISSA, International school for advanced studies, 34100 Trieste, Italy

${ }^{4}$ DEMOCRITOS, Modeling center for research in atomistic simulation, INFM, Italy

\section{Supporting Information}


The study of DSA-model N-acetyl-DSA (Figure 2) in vacuo presented in reference(1) is here extended to larger values of $\chi_{1}$ and $\chi_{2}$ : the geometry is optimized by quenching the structure while keeping one of the torsional angles fixed. The equilibrium values refer to the crystal structure of 2-Aza-4,4-spirocyclopropacycloohexadienone, which is structurally closely related to our model compound (coordinates are taken from the Cambridge database, ID: RUGGAB(2)). The structural and electronic differences are quantified in terms of bond lengths and Boys' Orbitals for eight selected conformations (Tab. S1). 
Table S1

A) Conformations for $\mathrm{N}$-acetyl-cyclopropylindole (Figure $\left.\left[{ }^{\circ}\right] . \mathrm{B}\right)$ Maximal changes observed in bond lengths $[\AA]$ and Boys Orbitals.

\begin{tabular}{|l|l|l|l|l|l|l|l|l|}
\hline A) & 1 & 2 & 3 & 4 & 5 & 6 & 7 \\
\hline$\chi 1$ & 7.0 & 7.0 & 7.0 & 7.0 & 0.0 & 10.0 & 20.0 \\
\hline$\chi 2$ & 5.0 & 10.0 & 20.0 & 30.0 & 5.0 & 5.0 & 5.0 \\
\hline
\end{tabular}

\begin{tabular}{|l|l|l|}
\hline B) & Bond-length & Wannier-Center \\
\hline C9-C10 & 0.007 & 0.004 \\
\hline C9-C13 & 0.005 & 0.001 \\
\hline C10-C13 & 0.003 & 0.001 \\
\hline N12-C16 & 0.007 & 0.006 \\
\hline N12-C8 & 0.004 & 0.004 \\
\hline C8-c7 & 0.003 & 0.015 \\
\hline
\end{tabular}


Table S2: MD simulations. (i) Local axis bend, (ii) roll angle and (iii) global curvature with standard deviations in parentheses for covalent drug-DNA adducts and free DNA.

(i) local axis bend

\begin{tabular}{|l|l|l|l|}
\hline & CovDSA・DNA & Cov-DSI •DNA & Free DNA \\
\hline A2-C3 & $3.7(1.6)$ & $4.0(1.6)$ & $3.5(1.6)$ \\
\hline C3-T4 & $2.8(1.7)$ & $4.8(2.1)$ & $3.7(2.1)$ \\
\hline T4-A5 & $4.8(2.4)$ & $3.8(1.8)$ & $6.0(2.8)$ \\
\hline A5-A6 & $3.9(2.0)$ & $3.6(1.1)$ & $3.1(2.2)$ \\
\hline A6-T7 & $4.0(2.1)$ & $3.6(1.9)$ & $4.1(2.0)$ \\
\hline T7-T8 & $3.8(2.0)$ & $6.0(2.4)$ & $5.7(2.5)$ \\
\hline T8-G9 & $6.6(3.0)$ & $2.9(1.7)$ & $3.3(1.7)$ \\
\hline G9-A10 & $3.2(2.0)$ & & \\
\hline
\end{tabular}

(ii) roll angle

\begin{tabular}{|l|l|l|l|}
\hline & CovDSA-•DNA & Cov-DSI-•DNA & Free DNA \\
\hline A2-C3 & $-0.3(4.6)$ & $0.1(4.0)$ & $3.5(1.6)$ \\
\hline C3-T4 & $1.2(4.8)$ & $10.5(6.0)$ & $3.7(2.1)$ \\
\hline T4-A5 & $11.2(6.2)$ & $-0.6(5.4)$ & $6.0(2.8)$ \\
\hline A5-A6 & $0.1(5.6)$ & $0.8(4.1)$ & $3.1(2.3)$ \\
\hline A6-T7 & $1.5(4.2)$ & $0.1(4.5)$ & $4.1(2.0)$ \\
\hline T7-T8 & $0.4(4.6)$ & $11.4(5.7)$ & $5.7(2.5)$ \\
\hline T8-G9 & $11.0(6.7)$ & $1.8(5.2)$ & $3.3(1.7)$ \\
\hline G9-A10 & $0.5(6.1)$ & & \\
\hline
\end{tabular}

(iii) global axis curvature $\left[{ }^{\circ}\right]$, first row: average values, in paranthesis standard deviations, second row: maximal variation along normal modes, in paranthesis corresponding normal mode.

\begin{tabular}{|l|l|l|}
\hline CovDSA•DNA & CovDSI•DNA & Free DNA \\
\hline $16.8(7.6)$ & $14.7(6.9)$ & $24.4(9.6)$ \\
\hline $13.7(\mathrm{NM} 2)$ & $14.6(\mathrm{NM} 1)$ & $10.3(\mathrm{NM} 2)(17.7, \mathrm{NM} 5)$ \\
\hline
\end{tabular}


Table S3: MD simulations. (i) Local Axis Bend, (ii) roll angle and (iii)global curvature with standard deviations in paranthesis for non-covalent adducts.

(i) local axis bend

\begin{tabular}{|l|l|l|l|}
\hline & DSA・DNA & DSI・DNA & NBOC-DSA・DNA \\
\hline A2-C3 & $3.6(1.7)$ & $4.1(2.0)$ & $3.5(1.7)$ \\
\hline C3-T4 & $4.1(2.1)$ & $4.5(2.5)$ & $4.1(2.2)$ \\
\hline T4-A5 & $7.0(3.4)$ & $6.8(3.8)$ & $7.6(3.6)$ \\
\hline A5-A6 & $3.4(1.8)$ & $3.3(1.7)$ & $4.4(2.6)$ \\
\hline A6-T7 & $3.0(1.6)$ & $3.3(1.9)$ & $3.9(2.2)$ \\
\hline T7-T8 & $3.8(1.9)$ & $5.7(2.5)$ & $4.1(2.1)$ \\
\hline T8-G9 & $5.9(2.4)$ & $2.8(1.6)$ & $5.9(2.7)$ \\
\hline G9-A10 & $3.2(1.8)$ & & $3.8(2.0)$ \\
\hline
\end{tabular}

(ii) roll angle

\begin{tabular}{|l|l|l|l|}
\hline & DSA•DNA & DSI•DNA & NBOC-DSA・DNA \\
\hline A2-C3 & $2.4(4.1)$ & $-0.41(5.3)$ & $3.5(1.7)$ \\
\hline C3-T4 & $4.9(5.2)$ & $13.0(7.3)$ & $4.1(2.2)$ \\
\hline T4-A5 & $11.7(7.6)$ & $1.6(6.0)$ & $4.6(3.6)$ \\
\hline A5-A6 & $-1.2(5.8)$ & $-1.6(3.9)$ & $3.9(2.2)$ \\
\hline A6-T7 & $-0.7(4.2)$ & $-2.7(4.7)$ & $4.1(2.1)$ \\
\hline T7-T8 & $0.0(4.8)$ & $11.9(5.2)$ & $5.9(2.7)$ \\
\hline T8-G9 & $12.0(5.4)$ & $-0.1(5.2)$ & $3.8(2.0)$ \\
\hline G9-A10 & $-1.3(4.7)$ & & \\
\hline
\end{tabular}

(iii) global axis curvature $\left[^{\circ}\right]$, first row: average values, in paranthesis standard deviations, second row: maximal variation along normal modes, in paranthesis corresponding normal mode.

\begin{tabular}{|l|l|l|}
\hline DSA・DNA & DSI•DNA & NBOC-DSA・DNA \\
\hline $17.7(7.7)$ & $15.3(8.7)$ & $29.4(10.1)$ \\
\hline $11.5(\mathrm{NM} 1)(22.0, \mathrm{NM} 4)$ & $14.5(\mathrm{NM} 1)(19.2, \mathrm{NM} 4)$ & $13.0(\mathrm{NM} 1)(19.7, \mathrm{NM} 4)$ \\
\hline
\end{tabular}


Table S4: MD simulations. Backbone parameters (i) $\gamma$, (ii) $\varepsilon$, (iii) $\zeta$, and (iv) $\chi$, for which experimentally conformational changes at alkylation site have been observed.

\begin{tabular}{|c|c|c|c|c|c|c|}
\hline (i) gamma $\gamma$ & DNA & DSA $\cdot$ DNA & DSI·DNA & $\begin{array}{l}\text { NBOC- } \\
\text { DSA•DNA }\end{array}$ & CovDSA・DNA & CovDSIDNA \\
\hline A2 & 51.2 & 49.1 & 51.8 & 50.2 & 48.6 & 52.1 \\
\hline $\mathrm{C} 3$ & 54.7 & 56.0 & 53.6 & 55.0 & 53.3 & 55.4 \\
\hline $\mathrm{T} 4$ & 54.8 & 53.6 & 72.8 & 55.5 & 57.2 & 55.5 \\
\hline T5 & 49.7 & 52.6 & 50.5 & 54.2 & 45.8 & 48.1 \\
\hline A6 & 49.0 & 49.0 & 46.9 & 56.6 & 44.1 & 42.3 \\
\hline A7 & 54.4 & 54.1 & 53.4 & 57.0 & 52.6 & 53.6 \\
\hline T8 & 53.3 & 55.1 & 53.7 & 54.1 & 55.1 & 55.0 \\
\hline G9 & 47.8 & 44.9 & 45.5 & 45.4 & 48.8 & 46.8 \\
\hline A10 & 45.5 & 47.5 & 43.4 & 43.0 & 43.9 & 41.8 \\
\hline T13 & 52.5 & 54.8 & 51.8 & 53.3 & 51.2 & 53.3 \\
\hline C14 & 53.3 & 52.5 & 53.1 & 52.6 & 53.5 & 53.8 \\
\hline A15 & 49.2 & 48.0 & 48.0 & 47.5 & 48.5 & 48.0 \\
\hline A16 & 47.9 & 45.2 & 43.1 & 48.4 & 48.5 & 45.4 \\
\hline T17 & 54.1 & 53.6 & 54.0 & 54.5 & 53.3 & 55.4 \\
\hline T18 & 53.3 & 59.3 & 56.1 & 53.4 & 59.4 & 57.2 \\
\hline A19 & 50.7 & 58.1 & 54.5 & 51.8 & 49.7 & 48.2 \\
\hline G20 & 47.3 & 51.5 & 46.7 & 52.1 & 49.4 & 46.4 \\
\hline $\mathrm{T} 21$ & 53.4 & 53.2 & 52.7 & 51.5 & 51.3 & 50.5 \\
\hline
\end{tabular}

\begin{tabular}{|l|l|l|l|l|l|l|}
\hline (ii) epsilon $\varepsilon$ & & & & & & \\
\hline A2 & $-167.0 / 174.3$ & $-170.8 / 173.3$ & $-170.0 / 172.9$ & $-160.0 / 173.4$ & $-159.1 / 172.3$ & $-167.1 / 172.6$ \\
\hline C3 & $/ 173.9$ & $-170.2 / 174.6$ & $-158.7 / 175.1$ & $-170.4 / 175.0$ & $-173.0 / 174.1$ & $-171.1 / 173.2$ \\
\hline T4 & $-164.0 / 175.4$ & $-159.5 / 175.7$ & $-155.8 / 175.7$ & $-168.0 / 175.0$ & $-170.4 / 172.1$ & $-168.6 / 173.5$ \\
\hline T5 & $-114.7 / 172.3$ & $-119.0 / 171.3$ & $-105.7 / 172.0$ & $-159.8 / 174.3$ & $-99.3 / 174.4$ & $-91.3 / 173.5$ \\
\hline A6 & $-177.9 / 173.2$ & $-172.9 / 173.6$ & $-172.9 / 174.2$ & $-170.9 / 173.9$ & $-171.9 / 174.4$ & $-171.1 / 173.7$ \\
\hline A7 & $-169.5 / 175.5$ & $-171.7 / 174.7$ & $-173.0 / 175.1$ & $-170.5 / 174.8$ & $-172.3 / 174.8$ & $-173.2 / 173.7$ \\
\hline T8 & $-162.0 / 175.1$ & $-164.6 / 175.2$ & $-163.5 / 175.7$ & $-161.5 / 176.6$ & $-165.3 / 175.5$ & $-164.5 / 173.8$ \\
\hline G9 & $-100.9 / 174.1$ & $-80.0 / 176.9$ & $-89.7 / 175.1$ & $-98.0 / 172.9$ & $-102.5 / 173.6$ & $-92.8 / 173.8$ \\
\hline A10 & $-170.9 / 174.4$ & $165.6 / 174.5$ & $-171.2 / 174.5$ & $-170.1 / 174.6$ & $-167.9 / 173.8$ & $-168.7 / 173.9$ \\
\hline T13 & $-161.7 / 174.5$ & $-166.9 / 175.7$ & $-156.8 / 174.6$ & $-155.0 / 174.2$ & $-95.1 / 148.0$ & $-167.1 / 174.6$ \\
\hline C14 & $/ 176.7$ & $-157.1 / 175.7$ & $-165.5 / 170.9$ & $-147.1 / 175.6$ & $-89.9 / 150.3$ & $-152.1 / 173.9$ \\
\hline A15 & $-110.1 / 172.8$ & $-97.7 / 171.5$ & $-83.8 / 170.9$ & $-124.5 / 173.9$ & $-101.4 / 144.2$ & $-95.9 / 173.6$ \\
\hline A16 & $-141.4 / 173.1$ & $-171.2 / 171.5$ & $-172.9 / 173.6$ & $-171.7 / 173.9$ & $-95.5 / 143.2$ & $-172.6 / 173.5$ \\
\hline T17 & $-170.8 / 175.3$ & $-169.8 / 175.1$ & $-169.8 / 175.8$ & $-169.2 / 175.1$ & $-93.1 / 155.4$ & $-170.4 / 175.8$ \\
\hline
\end{tabular}




\begin{tabular}{|l|l|l|l|l|l|l|}
\hline T18 & $-162.5 / 174.7$ & $-168.7 / 174.9$ & $-170.3 / 175.0$ & $-158.7 / 175.7$ & $-85.3 / 165.6$ & $-168.4 / 175.5$ \\
\hline A19 & $-115.3 / 173.3$ & $-151.1 / 175.0$ & $-133.8 / 173.4$ & $-133.8 / 173.4$ & $-110.4 / 149.5$ & $-92.7 / 173.8$ \\
\hline G20 & $-172.2 / 173.9$ & $-171.4 / 173.9$ & $-160.2 / 173.4$ & $-172.6 / 173.6$ & -93.9 & $-171.9 / 173.8$ \\
\hline T21 & $-166.8 / 175.3$ & $-166.9 / 174.2$ & $-159.0 / 174.8$ & $-167.7 / 174.8$ & -89.9 & $-168.5 / 173.2$ \\
\hline
\end{tabular}

\begin{tabular}{|c|c|c|c|c|c|c|}
\hline (iii) zeta $\zeta$ & & & & & & \\
\hline $\mathrm{A} 2$ & -93.7 & $-93.8 / 145.0$ & $-91.2 / 160.5$ & $-93.4 / 141.6$ & $-92.6 / 144.2$ & $-91.9 / 142.2$ \\
\hline C3 & -89.7 & -92.1 & $-93.8 / 151.7$ & -90.7 & -92.6 & $-90.5 / 142.4$ \\
\hline $\mathrm{T} 4$ & $-97.3 / 148.0$ & $-89.2 / 157.4$ & $-82.4 / 157.4$ & $-82.4 / 140.0$ & $-89.2 / 171.8$ & $-87.2 / 148.6$ \\
\hline T5 & $-102.3 / 145.0$ & $-102.5 / 146.8$ & $-100.3 / 144.3$ & $-96.5 / 156.3$ & $-118.7 / 150.5$ & $-104.5 / 144.0$ \\
\hline A6 & -94.6 & $\begin{array}{l}-91.2 \\
\end{array}$ & -91.2 & -88.7 & -90.3 & $-90.2 / 144.0$ \\
\hline A7 & -90.0 & -88.0 & -90.6 & -91.8 & -90.3 & $-90.7 / 144.0$ \\
\hline T8 & $-85.0 / 143.0$ & -82.0 & -82.1 & $-87.1 / 152.4$ & -90.3 & $-81.9 / 144.2$ \\
\hline G9 & $-107.6 / 143.0$ & $-150.8 / 137.7$ & $-127.0 / 143.2$ & $-111.2 / 143.1$ & $-83.0 / 154.5$ & $-114.8 / 143.3$ \\
\hline A10 & -88.2 & -84.6 & -89.2 & -88.0 & -106.3 & $-89.2 / 147.3$ \\
\hline T13 & $-95.0 / 142.2$ & -91.6 & $-95.4 / 145.8$ & $-92.9 / 144.5$ & $-90.3 / 138.8$ & $-94.8 / 152.3$ \\
\hline C14 & $-91.7 / 150.1$ & $-88.3 / 158.2$ & -85.7 & $-95.1 / 152.0$ & $-89.9 / 150.3$ & $-88.7 / 144.5$ \\
\hline A15 & $-104.0 / 143.5$ & $-103.1 / 141.4$ & $-129.3 / 141.9$ & $-101.4 / 146.7$ & $-101.4 / 144.2$ & $-101.3 / 144.5$ \\
\hline A16 & -92.5 & $\begin{array}{l}-91.6 \\
\end{array}$ & -92.7 & -93.6 & $-95.5 / 143.2$ & $-93.4 / 169.1$ \\
\hline T17 & -91.1 & $-94.9 / 144.8$ & -91.9 & $-90-2$ & $-93.1 / 155.4$ & -91.7 \\
\hline T18 & $-89.3 / 163.8$ & -77.0 & -80.1 & $-91.2 / 157.6$ & $-85.3 / 165.6$ & $-82.3 / 167.3$ \\
\hline A19 & $-112.2 / 147.8$ & $-105.5 / 158.2$ & $-108.3 / 158.5$ & $-100.2 / 150.8$ & $-110.4 / 149.5$ & $-115.2 / 146.7$ \\
\hline G20 & -94.3 & -93.0 & $-94.1 / 136.6$ & -97.2 & -93.9 & -90.7 \\
\hline $\mathrm{T} 21$ & -89.8 & $-87.0 / 149.9$ & $-86.8 / 145.6$ & -90.4 & -89.9 & -88.0 \\
\hline
\end{tabular}

\begin{tabular}{|l|l|l|l|l|l|l|}
\hline (vi) chi $\chi$ & & & & & & \\
\hline A2 & -64.1 & -65.3 & -65.9 & -68.3 & -64.3 & -63.9 \\
\hline C3 & -66.0 & -66.6 & -71.8 & -68.4 & -60.9 & -64.0 \\
\hline T4 & -71.8 & -71.3 & -77.3 & -66.6 & -68.0 & -66.0 \\
\hline T5 & -73.7 & -67.7 & -71.3 & -64.3 & -72.4 & -72.3 \\
\hline A6 & -63.5 & -62.2 & -63.4 & -62.0 & -65.0 & -65.0 \\
\hline A7 & -65.6 & -61.6 & -59.3 & -64.7 & -62.5 & -59.4 \\
\hline T8 & -65.6 & -73.8 & -74.8 & -73.8 & -71.2 & -74.2 \\
\hline G9 & -73.3 & -38.8 & -82.9 & -79.5 & -77.0 & -80.7 \\
\hline A10 & -79.0 & -70.7 & -68.8 & -67.8 & -66.9 & -68.0 \\
\hline T13 & -67.8 & -68.5 & -66.1 & -68.8 & -70.2 & -66.9 \\
\hline C14 & -67.3 & -75.7 & -75.0 & -73.5 & -73.5 & -75.7 \\
\hline A15 & -73.8 & -77.2 & -83.5 & -73.7 & -73.4 & -78.3 \\
\hline
\end{tabular}




\begin{tabular}{|l|l|l|l|l|l|l|}
\hline A16 & -76.0 & -66.1 & -63.9 & -64.2 & -63.7 & -61.7 \\
\hline T17 & -63.5 & -65.1 & -66.8 & -67.6 & -66.5 & -66.1 \\
\hline T18 & -69.8 & -63.2 & -64.5 & -70.1 & -66.8 & -69.4 \\
\hline A19 & -74.3 & -64.9 & -68.9 & -69.0 & -72.3 & -76.5 \\
\hline G20 & -65.2 & -63.7 & -64.5 & -63.8 & -64.4 & -64.5 \\
\hline T21 & -68.5 & -68.2 & -69.8 & -70.2 & -67.5 & -66.6 \\
\hline
\end{tabular}


Table S5: Hybrid Car-Parrinello QM/MM simulations. Torsional angles (in degrees) in the reactions of DSA, DSI and NBOC-DSA with DNA and NBOC-DSA with adenine. Standard deviations given in parenthesis.

\begin{tabular}{|c|c|c|c|c|}
\hline$\chi_{1}$ & DSA・DNA & DSI•DNA & NBOC-DSA・DNA & NBOC-DSA•adenine \\
\hline 3.0 & $22(8)$ & $12(6)$ & $24(10)$ & $24(8)$ \\
\hline 2.8 & $17(16)$ & $16(9)$ & $26(9)$ & $27(8)$ \\
\hline 2.6 & $13(10)$ & $20(7)$ & $26(8)$ & $22(10)$ \\
\hline 2.4 & $14(6)$ & $12(5)$ & $17(8)$ & $13(7)$ \\
\hline 2.3 & $11(8)$ & $2(11)$ & $4(7)$ & $12(10)$ \\
\hline \multicolumn{5}{|l|}{$\chi_{2}$} \\
\hline 3.0 & $13(6)$ & $17(7)$ & $-6(8)$ & $-17(14)$ \\
\hline 2.8 & $17(6)$ & $6(6)$ & $-7(7)$ & $-13(7)$ \\
\hline 2.6 & $11(6)$ & $17(7)$ & $-11(8)$ & $-5(3)$ \\
\hline 2.4 & $11(6)$ & $16(6)$ & $-4(7)$ & $2(8)$ \\
\hline
\end{tabular}

Table S6: B3LYP geometry optimization of N-Acetyl-cyclopropylindole in presence of at $\mathrm{d}(\mathrm{C} 13-$ $\left.\mathrm{N}\left(\mathrm{NH}_{3}\right)\right)=2.8,2.6,2.4$ and 2.2 for $\mathrm{R} 2$ at B3LYP level in vacuo and in vacuo and with implicit solvent (PCM). The optimized geometries of B3LYP are used to estimate energies at the BLYP and/or with implicit solvent (PCM). The Gaussian program has been used.

\begin{tabular}{|l|l|l|l|l|}
\hline & B3LYP & BLYP & B3LYP (PCM) & BLYP (PCM) \\
\hline$\Delta \mathrm{Ea}[\mathrm{kcal} / \mathrm{mol}]$ & 18.8 & 16.4 & 9.6 & 6.3 \\
\hline
\end{tabular}




\section{Reference List}

1. Kirschner K.N., Lee M., Stanley R.C. and Bowen J.P. Density Functional and Ab Initio Studies on N-Acetyl-Duocarmycin SA: Insight Into Its DNA Interaction Properties (2000) Bioorg.Med.Chem., 8, 329-335.

2. Parrish J.P., Kastrinsky D.B. and Boger D.L. Synthesis and X-ray Analysis of an Unprecedented and Stable 2-Aza-4,4-spirocyclopropacyclohexadienone (2003) Org.Lett., 5, 2577-2579. 


\section{Figure Captions}

Figure S1: Left: Superposition of NMR structures of covDSA•DNA (blue) and covDSI•DNA (red). The drugs are shown in ball and sticks and the reactive adenine residue (A19) in cylinders. Right: Solvent accessible surface of the double helix with electrostatic color code and DSI and DSA in ball and sticks inside the minor groove.

Figure S2: Model system N-acetyl cyclopropylindole, for which torsion angle profile (Table S1) and test calculations with B3LYP have been calculated (Table S6).

Figure S3: Upper four graphs: rmsds for free DNA and non-covalent drug-DNA adducts. Lower three graphs: N3(A19)-C13 distance [nm] in black and N3(G20)-C13 distance [nm] in red for the non-covalent drug-DNA complexes.

Figure S4: Changes in minor groove width along the first three normal modes at the central steps is shown as bars. The extent of the bar indicates the total variation along the normal mode. Black: Free DNA, Red: DSA•DNA, blue: DSI•DNA, violet: NBOC-DSA•DNA. (DNA is abbreviated with I)

Figure S5: Variation of local axis bend at step 4 (T4-A5) and step 8 (T8-G9) along the first five normal modes in the non-covalent (upper panel) and in the covalent adducts (lower panel). Black: Free DNA, Red: DSA・DNA blue: DSI•DNA, violet: NBOC- DSA・DNA . (DNA is abbreviated with I)

Figure S6: Torsional angle distribution for non-covalent drug-DNA adducts. Solid line: classical molecular dynamics simulation, Dashed lines: QM/MM simulation, entire trajectory. 

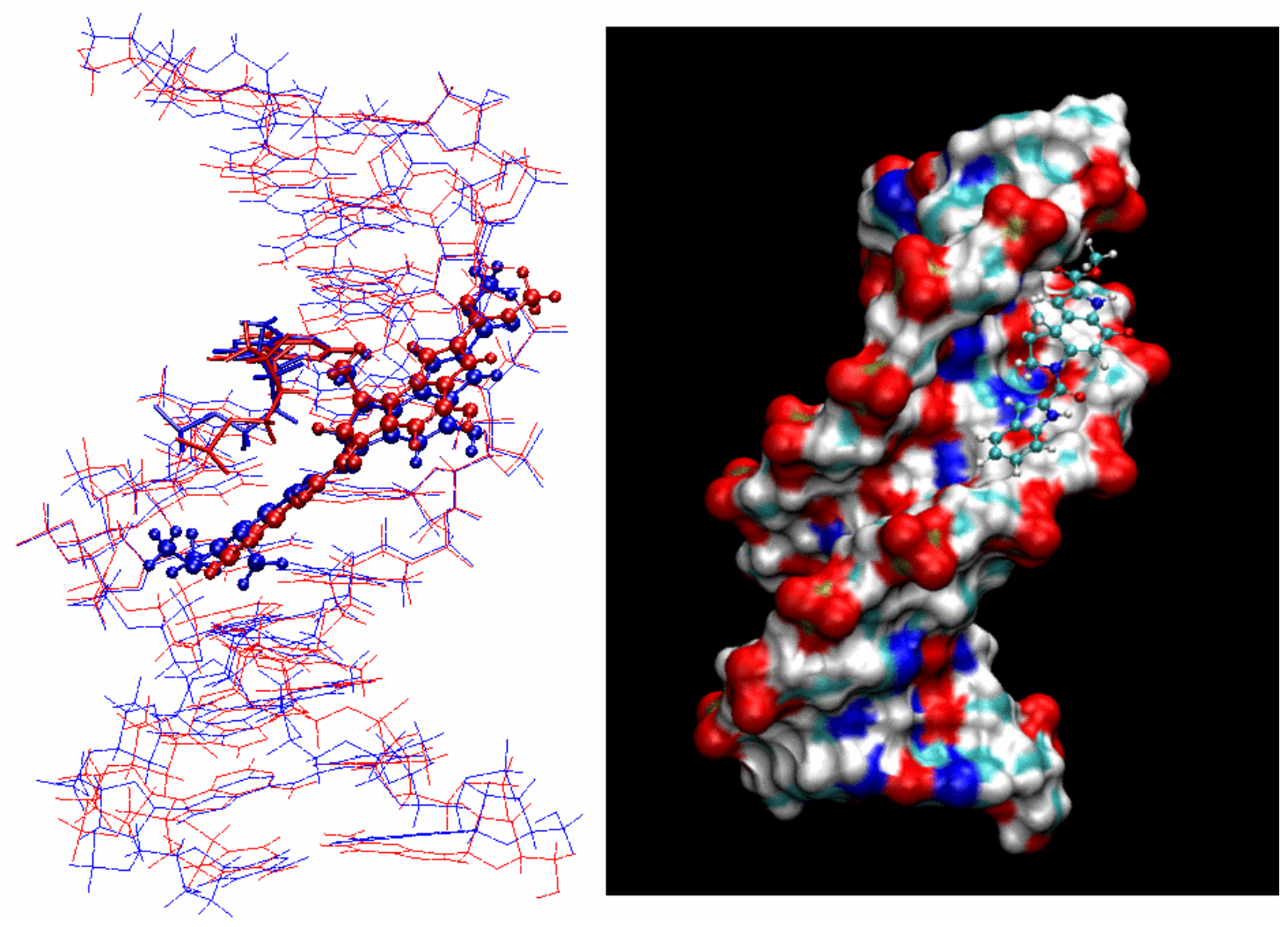

Figure S1

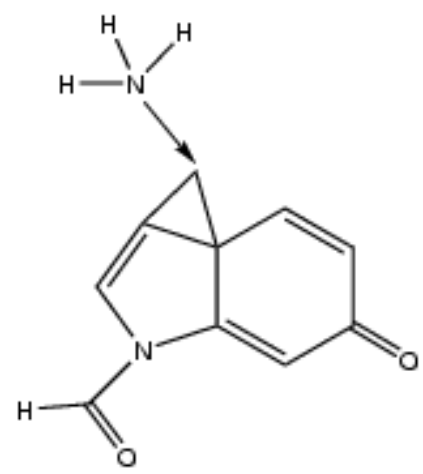

Figure S2 

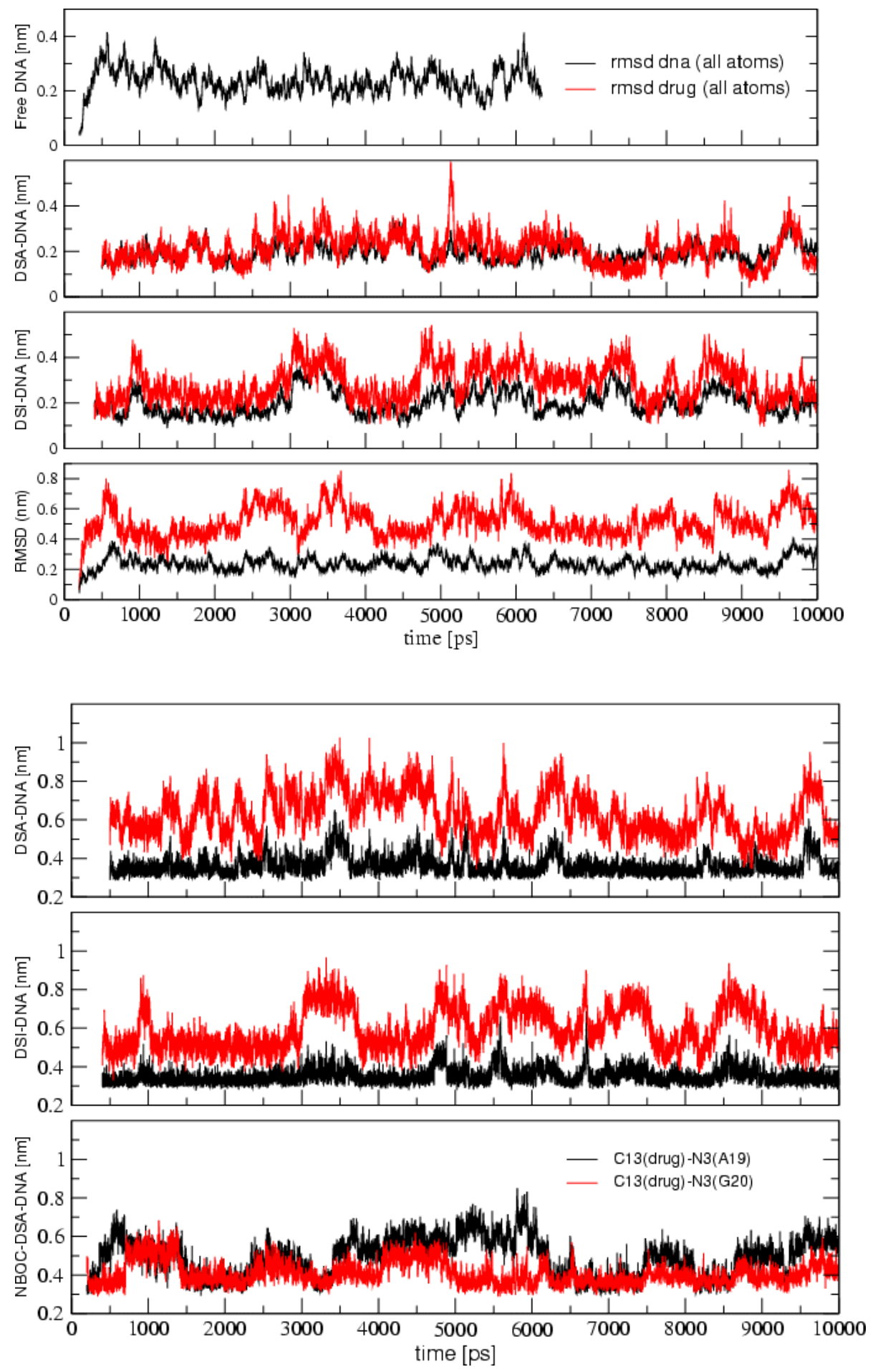

Figure S2 

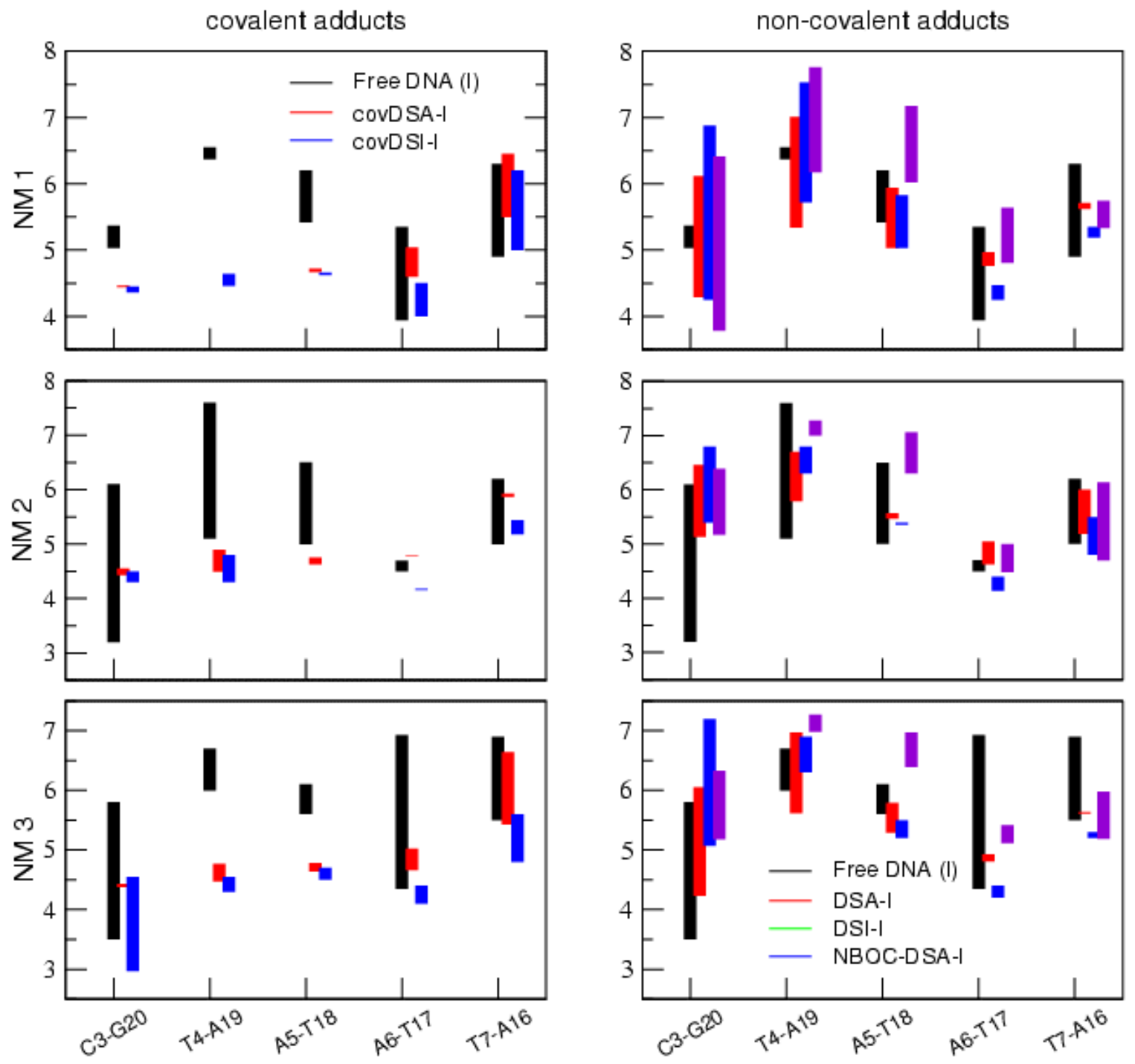

Figure S3 
T4-A5
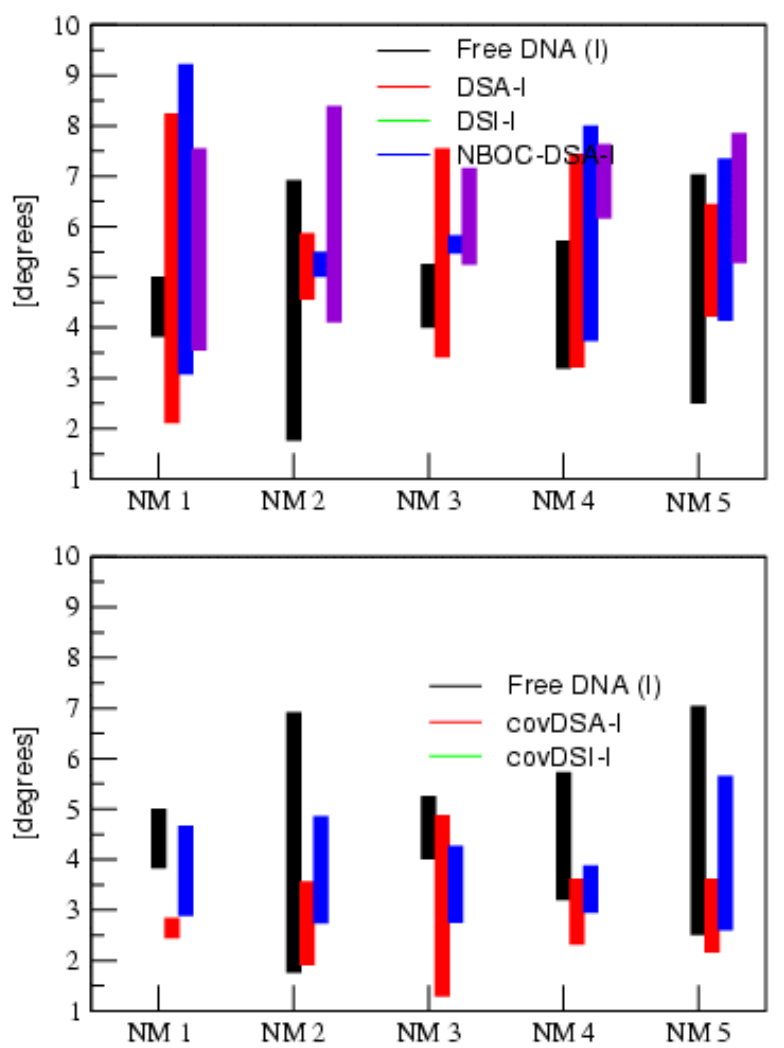

T8-G9
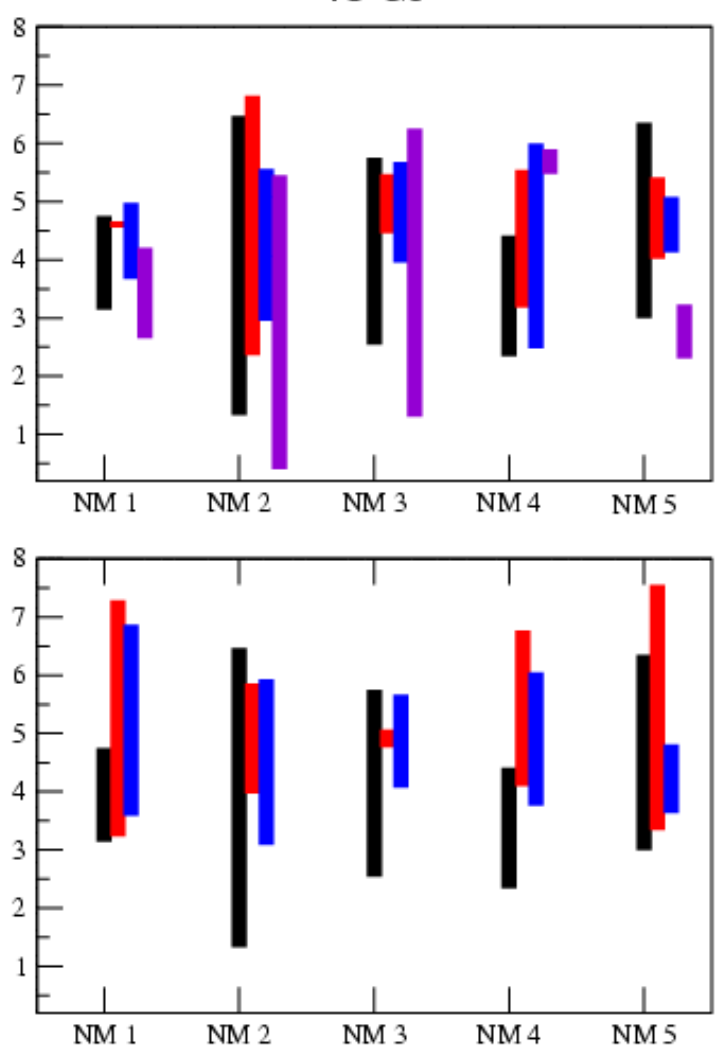

Figure S4 

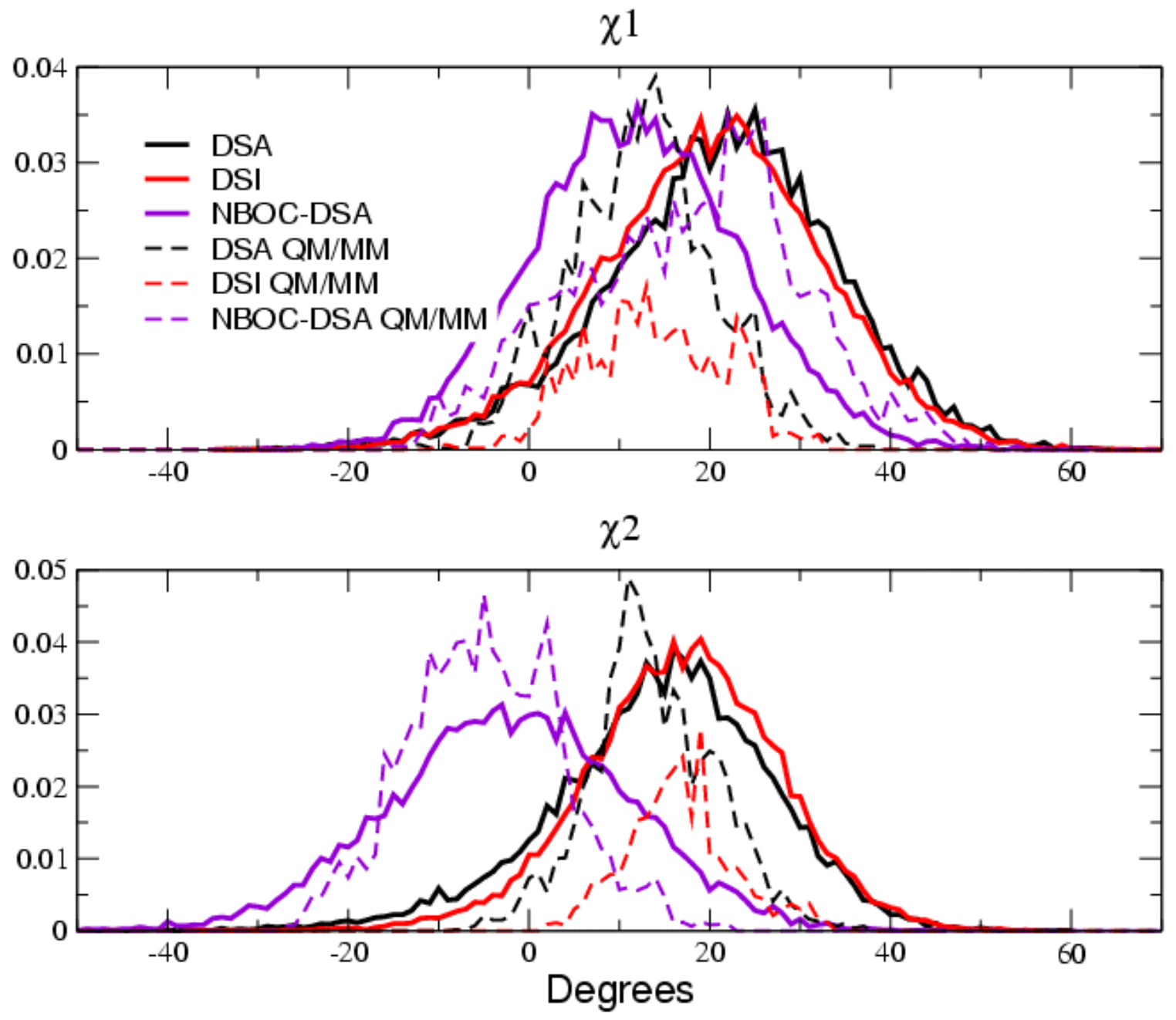

Figure S5 